\title{
Synergies and complementarities between proposed future neutrino projects
}

Sushant K. Raut*

Center for Theoretical Physics of the Universe, Institute for Basic Science (IBS), Daejeon, 34051, Korea

E-mail: sushanteibs.re.kr

\begin{abstract}
Measuring the unknown neutrino oscillation parameters is one of the main aims in neutrino physics today. The measurement of these parameters is severely affected by the presence of degeneracies in the parameter space. Various neutrino oscillation projects have been proposed to measure them. In this overview talk, we will discuss some of the proposed neutrino experiments and the synergies between them that can enhance their future physics reach.
\end{abstract}

The 19th International Workshop on Neutrinos from Accelerators-NUFACT2017

25-30 September, 2017

Uppsala University, Uppsala, Sweden

\footnotetext{
* Speaker.
} 


\section{General framework}

The discovery of neutrino oscillations and hence of non-zero neutrino masses was the first sign of a deviation from the canonical Standard Model. Over the last two decades, neutrino oscillation physics has increasingly been seen as a window to new physics, in particular on models that could explain neutrino masses and mixing. While the mechanism of neutrino oscillations does not depend directly on these models, the values of the oscillation parameters can be useful discriminators between competing models.

In the standard three-flavour framework, neutrino oscillation probabilities depend on three mixing angles $-\theta_{12}, \theta_{13}$ and $\theta_{23}$, two mass-squared differences between the neutrino mass eigenstates $-\Delta m_{21}^{2}$ and $\Delta m_{31}^{2}$, and the CP-violating phase $\delta_{C P}$. Solar and reactor neutrino experiments have already measured $\theta_{12}, \theta_{13}$ and $\Delta m_{21}^{2}$ to very good precision. According to recent global fits [1], the magnitude of $\Delta m_{31}^{2}$ is known to a few percent precision but its sign (positive, called normal hierarchy $(\mathrm{NH})$ or negative, called inverted hierarchy $(\mathrm{IH})$ ) is not known. The octant of the close-to-maximal mixing angle $\theta_{23}\left(<45^{\circ}\right.$, lower octant (LO) or $>45^{\circ}$, higher octant (HO)) is unknown. The value of the phase $\delta_{C P}$ is also largely unknown at present. In addition, if extra sterile neutrino states or non-standard interactions (NSIs) exist in nature, they may introduce more parameters that are yet to be measured.

In this overview talk, we will discuss the parameter degeneracies that affect the measurement of the above parameters (primarily within the standard oscillation framework). We will see how various proposed experiments can achieve these measurements, singly and through a synergistic combination with other facilities. (Disclaimer: The list of references cited in this proceedings article are chosen to highlight some physics points and must not be considered exhaustive.)

\section{Parameter degeneracies}

The $v_{\mu} \rightarrow v_{e}$ oscillation channel is sensitive to all three unknown parameters and hence the probability $P_{\mu e}$ is useful for understanding the reach of various beam-based and atmospheric neutrino experiments. These experiments measure event rates which depend on $P_{\mu e}$ and hence the unknown parameters. It is possible for multiple combinations of these unknown parameters to give the same oscillation probability. This is the problem of parameter degeneracy [2].

For instance, the enhancement in $P_{\mu e}$ due to matter effects in case of $\mathrm{NH}$ can be offset if $\delta_{C P}$ is close to $90^{\circ}$. Similarly, the decrease in $P_{\mu e}$ due to matter effects in case of IH can be offset if $\delta_{C P}$ is close to $-90^{\circ}$. Therefore the combination of $\mathrm{NH}$ and $\delta_{C P}$ in the upper half-plane (UHP) is degenerate with the combination of IH and $\delta_{C P}$ in the lower half-plane (LHP) [3, 4]. This degeneracy is the same for antineutrinos. For the octant, it is found that the hierarchy-octant combination of $\mathrm{NH}$ and $\mathrm{LO}$ is degenerate with $\mathrm{IH}$ and $\mathrm{HO}$ in case of neutrinos, while $\mathrm{NH}$ and $\mathrm{HO}$ is degenerate with IH and LO for antineutrinos [5]. All these features can be explained using the approximate analytic expressions for $P_{\mu e}[6]$.

Figures 1 and 2 represent these degeneracies schematically for neutrinos and antineutrinos, respectively. In each figure, the left(right) part represents the parameter space for $\mathrm{IH}(\mathrm{NH})$. Within each of these parts, the inner(outer) ring corresponds to the choice of octant $\mathrm{LO}(\mathrm{HO})$. In each ring, the angular direction gives the value of $\delta_{C P}$. The $\delta_{C P}$ parameter space is divided into two half- 
planes LHP and UHP. Thus the complete parameter space in each figure is divided into eight parts, corresponding to the two choices of hierarchy, octant and half-plane of $\delta_{C P}$.

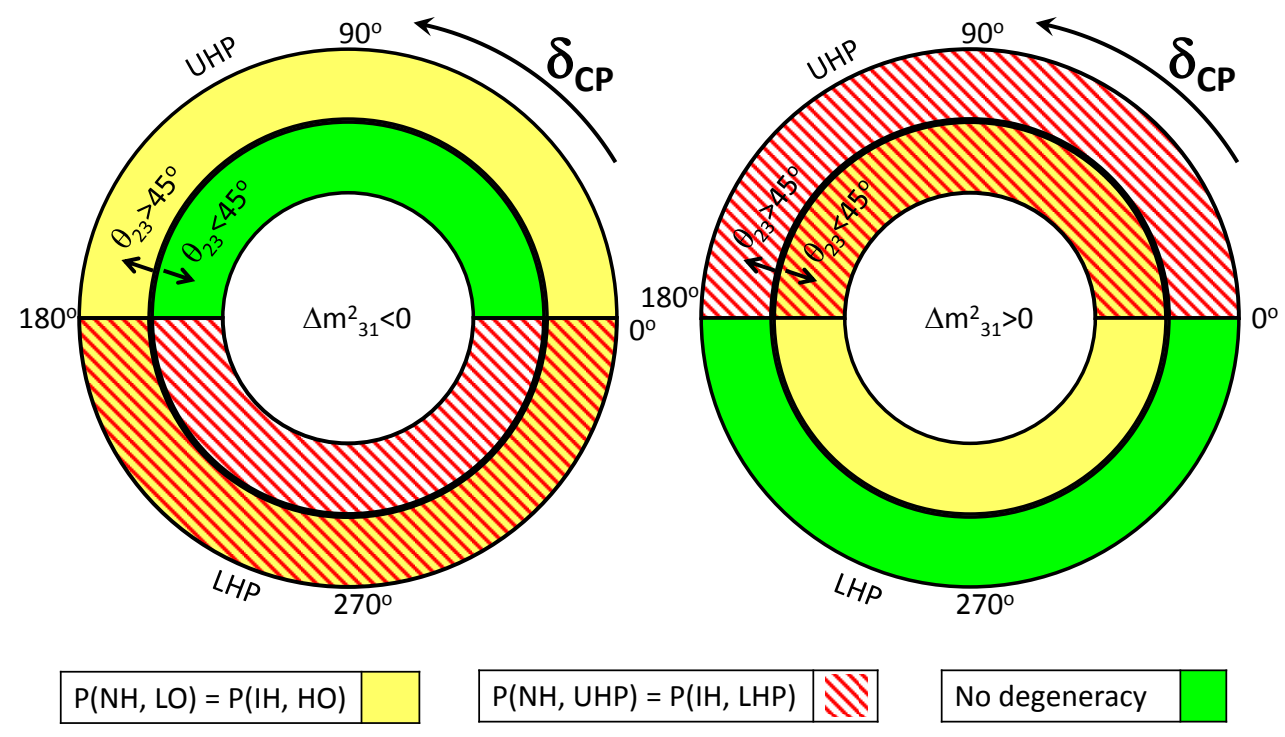

Figure 1: Schematic representation of parameter degeneracies in three-flavour neutrino oscillations. See the text for a detailed description.

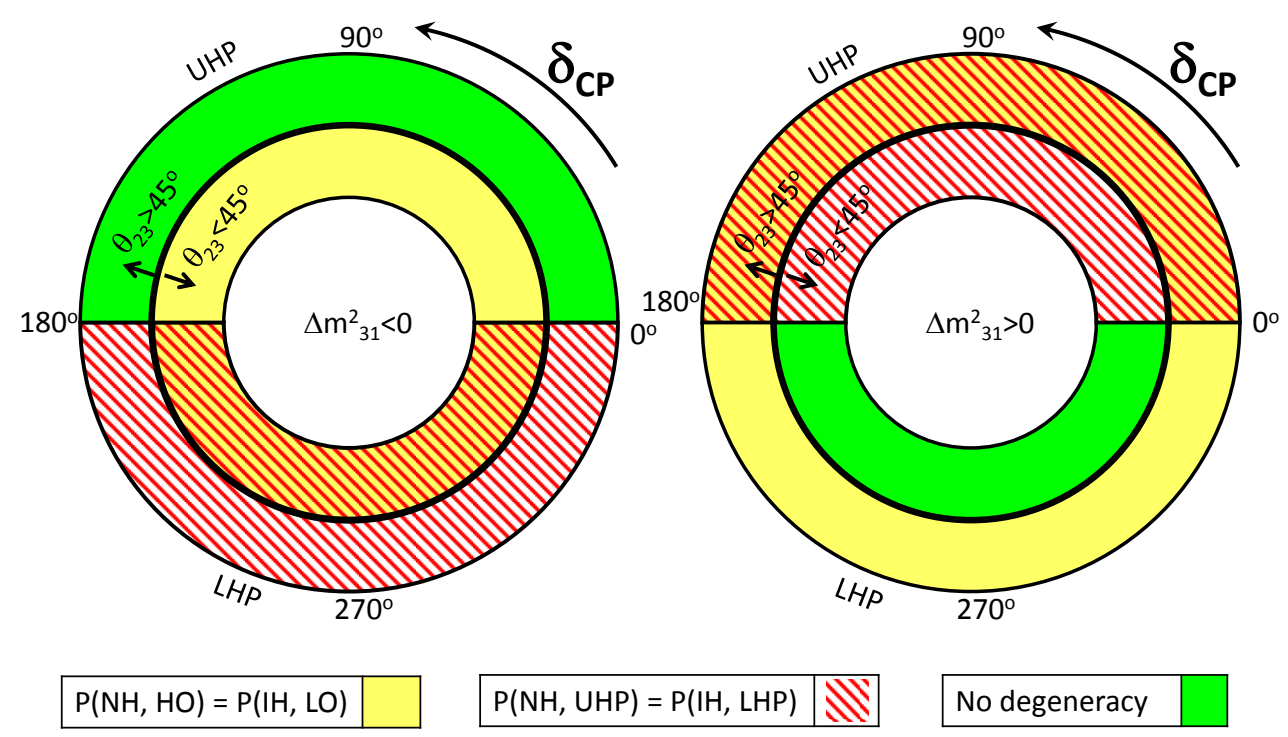

Figure 2: Same as Fig. 1, but for antineutrinos.

Next, we shade the parts of this parameter space that are degenerate with each other, as indicated in the legend. The octant degeneracy is coloured yellow while the hierarchy- $\delta_{C P}$ degeneracy is shaded with red stripes. The parts of the diagram that are left unshaded are marked in green and are free of parameter degeneracies. If the values of parameters in nature lie in the green region of 
Fig. 1, the neutrino run of the current generation of experiments will measure them without being hampered by degeneracies. If they lie in the yellow solid region, collecting data with antineutrinos will break the octant degeneracy since it affects neutrinos and antineutrinos in opposite ways (compare Figs. 1 and 2). However, if the parameters lie in the striped region, switching polarities does not help to lift the hierarchy degeneracy.

\section{Proposed future neutrino projects}

Some of the proposed experiments that will aim to measure the parameters are the longbaseline superbeam experiments DUNE [7], T2HK [8], T2HKK [9] and ESS $v$ SB [10], the mediumbaseline reactor neutrino experiment JUNO [11], the atmospheric neutrino experiments HK [12], ICAL@INO [13] and Deep Core/PINGU [14], and experiments based on a muon decay source, either in-flight like MOMENT [15] or at rest [16].

Each experiment has its characteristic baseline and energy, and hence has a different functional dependence on the oscillation parameters. Thus the degeneracies experienced by them are, in general, different. While all the experiments will point to the same correct set of parameters, they can help to exclude each others fake degenerate solutions. This synergy between the experiments will allow us to measure the oscillation parameters unambiguously, even if the individual experiments suffer from degeneracies [17].

\section{Measurement of unknown parameters}

First, we address the issue of determining the neutrino mass hierarchy. As discussed before, this can be impeded by the presence of the hierarchy- $\delta_{C P}$ degeneracy, if the combination of parameters in nature is unfavourable. In that case, in addition to the current generation of experiments, we need information from an experiment that has (a) more matter effects (in order to break the degeneracy), or (b) is insensitive to $\delta_{C P}$ (so that the hierarchy can be measured independently), or (c) has negligible matter effects (allowing $\delta_{C P}$ to be measured independently, thus lifting the degeneracy).

(a) The degeneracy is broken with more matter effects by future long-baseline experiments such as DUNE (which will exclude the wrong hierarchy at at least $3 \sigma$ even for unfavourable parameter values) [18]. The T2HKK proposal which aims to locate half of the HK detector in Korea in the path of the T2HK beam will combine data from the shorter T2HK and longer Tokai-to-Korea baselines. The addition of the longer-baseline component with more matter effects will increase the hierarchy sensitivity in the least favourable region from around $1 \sigma$ to $6 \sigma$ [9].

(b) Experiments that are insensitive to $\delta_{C P}$ such as atmospheric neutrino experiments can also determine the mass hierarchy, individually or in combination with long-baseline experiments. This has been illustrated in, for example, Refs. [19] and [20] in the context of ICAL and PINGU. The $\delta_{C P}$-independent hierarchy sensitivity of the atmospheric neutrino experiment pushes the combined sensitivity of the long-baseline+atmospheric experiments to higher confidence levels [18, 21].

(c) Experiments such as the muon decay-at-rest facilities with very short baselines are capable of measuring $\delta_{C P}$ accurately, because there is no interference between the CP and matter effects. These have traditionally been studied in the context of CP-measurements, but can also improve the 
mass hierarchy sensitivity [22]. In spite of having negligible hierarchy sensitivity by themselves, these experiments help by excluding the fake degenerate CP solution of long-baseline experiments, demonstrating remarkable synergy between the two.

It is also worth mentioning that the JUNO experiment aims to determine the mass hierarchy using a completely different method, i.e. by resolving the difference between the solar scale oscillations in case of $\mathrm{NH}$ and $\mathrm{IH}$ [11]. The confidence level at which the two can be distinguished depends on the energy resolution of the experiment.

Next, we discuss the measurement of $\delta_{C P}$. Once again, we can classify experiments into the three above categories. Long-baseline experiments with more matter effects can break the degeneracy between the unfavourable regions for $\mathrm{NH}$ and $\mathrm{IH}$ and determine the correct hierarchy, thus picking out the correct $\delta_{C P}$ solution. This results in an enhanced CP discovery potential (see for example Refs. [18] for DUNE and [9] for T2HKK) as well as a precise measurement of $\delta_{C P}$ [23]. Atmospheric neutrino experiments that are insensitive to $\delta_{C P}$ on their own can exclude the wrong hierarchy solution and increase the $\mathrm{CP}$ precision in conjunction with the current generation of long-baseline experiments [24]. Finally, experiments with small matter effects are ideal for measuring $\delta_{C P}$ without matter-induced fake CP-violation effects. These include muon decay-atrest experiments [25]. Another example is ESSvSB [26] which aims to measure $\delta_{C P}$ at the second oscillation maximum, where the CP-sensitivity $\mathrm{d} P_{\mu e} / \mathrm{d} \delta_{C P}$ is three times more than at the first oscillation maximum. Prototype-neutrino-factory experiments like MOMENT can also measure $\delta_{C P}$ in a synergistic combination with current experiments [27].

As discussed before, we need both neutrino and antineutrino data to determine the octant of $\theta_{23}$. DUNE and T2HK which will run with both polarities will measure the octant if $\theta_{23}$ is not very close to $45^{\circ}$. Atmospheric neutrino experiments like HK, ICAL and PINGU have also been shown to have good sensitivity to the octant. It is important to note that the effective atmospheric mixing angle measured by experiments is given by $\sin ^{2} 2 \theta_{\mu \mu}=4\left|U_{\mu 3}\right|\left(1-\left|U_{\mu 3}\right|^{2}\right)$, which is a function of both $\theta_{23}$ and $\theta_{13}$. Thus, the synergy between long-baseline/atmospheric neutrino experiments and reactor neutrino experiments is important in this case, i.e. a precise measurement of $\theta_{13}$ is crucial.

Finally we make a few comments on physics beyond the standard three-flavour scenario. Addition of extra sterile neutrinos introduces more mass-squared difference, mixing angles and $\mathrm{CP}$ phases that can affect the oscillations and create new degeneracies. The existence of chargedcurrent and neutral-current NSIs can also dramatically increase the dimension of the oscillation parameter space. In such cases, it is important to ask whether (a) the presence of these new parameters can affect the measurement of the standard oscillation parameters, and (b) our proposed experimental facilities can measure these new parameters. (While the presence of these large numbers of new parameters can seem overwhelming, it is important to remember that the search for these parameters is an opportunity to probe physics beyond the Standard Model, similar to collider searches.)

Given the large number of new parameters, it is difficult to make generalized statements about the degeneracies and their resolution in these new physics scenarios. A few specific cases have been studied in the literature. For instance, Ref. [28] discusses the octant- $\delta_{C P}-\delta_{14}$ degeneracy in the $3+1$ neutrino framework, which is hard to resolve even with a combination of neutrino and antineutrino data [29]. Depending on the true values of the parameters, experiments like DUNE and T2HKK may be able to resolve some of these degeneracies [30]. 
In the case of charged-current NSIs which are already well-bounded, experiments with small matter effects like ESS $v$ SB can probe the new parameters [31]. Neutral-current NSIs on the other hand are not constrained strongly at present, and can influence our measurements. Combinations of experiments such as DUNE with T2HK [32] and T2HKK [33] can help in some cases. To conclude, case-by-case studies of the various new physics scenarios are needed in order to formulate a strategy to deal with the new parameters.

\section{Summary}

We summarize with the help of this flowchart that shows a (very simplistic) roadmap for future oscillation studies. If the future oscillation data are found to be consistent with the standard oscillation framework, one can address the degeneracies (if they exist) by using data from different experiments. The synergies between various experiments on account of their different baseline and energy dependence will help to measure the parameters unambiguously. However if new physics effects are found in these data, more detailed studies will be required to devise methods to address the problems they will pose.

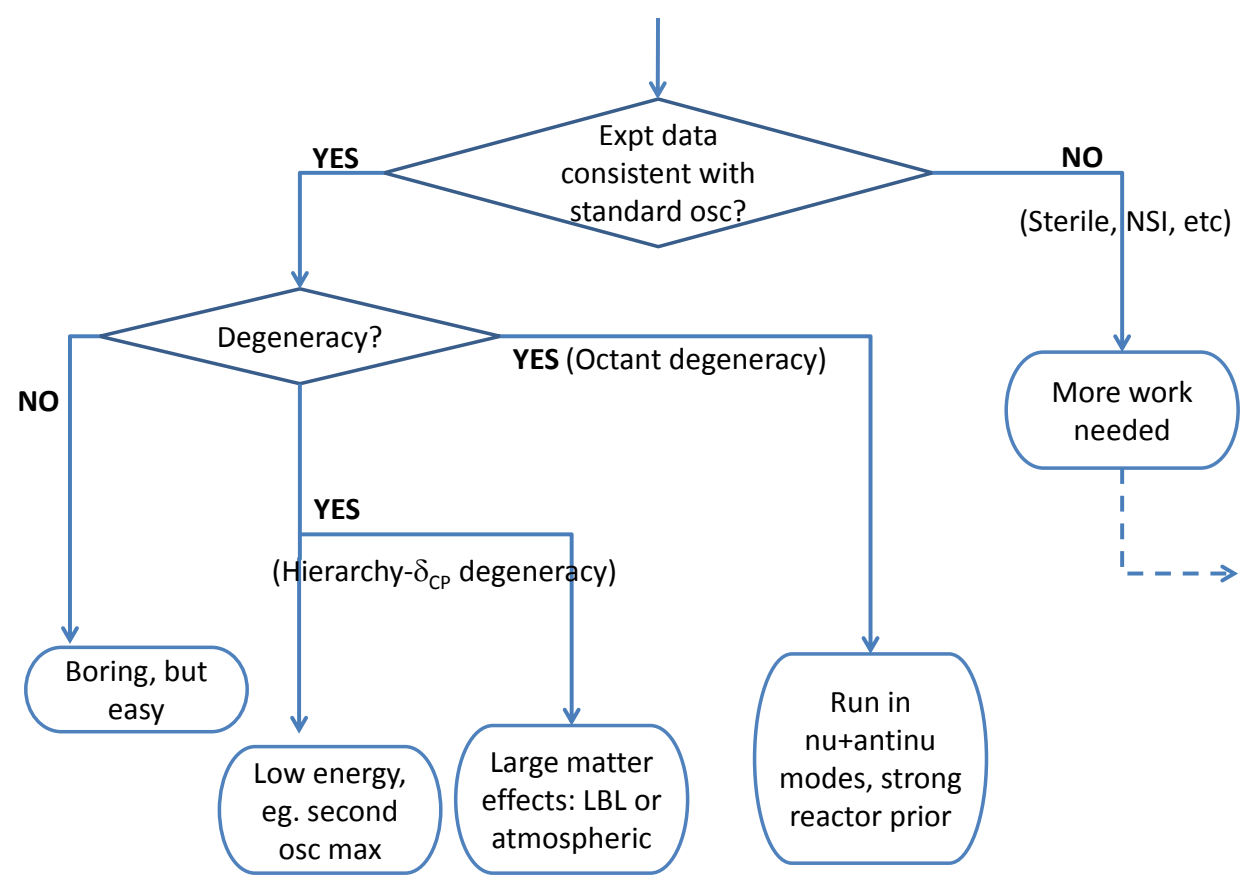

\section{References}

[1] F. Capozzi, E. Di Valentino, E. Lisi, A. Marrone, A. Melchiorri, and A. Palazzo, Global constraints on absolute neutrino masses and their ordering, arXiv:1703.04471.

[2] V. Barger, D. Marfatia, and K. Whisnant, Breaking eight-fold degeneracies in neutrino CP violation, mixing, and mass hierarchy, Phys. Rev. D65 (2002) 073023, [hep-ph/ 0112119$].$

[3] S. Prakash, S. K. Raut, and S. U. Sankar, Getting the Best Out of T2K and NOvA, Phys.Rev. D86 (2012) 033012, [arXiv:1201.6485]. 
[4] S. K. Agarwalla, S. Prakash, S. K. Raut, and S. U. Sankar, Potential of optimized NOvA for large $\theta_{(13)}$ and combined performance with a LArTPC and T2K, JHEP 1212 (2012) 075, [arXiv:1208.3644].

[5] S. K. Agarwalla, S. Prakash, and S. U. Sankar, Resolving the octant of theta23 with T2K and NOvA, JHEP 1307 (2013) 131, [arXiv:1301.2574].

[6] E. K. Akhmedov, R. Johansson, M. Lindner, T. Ohlsson, and T. Schwetz, Series expansions for three flavor neutrino oscillation probabilities in matter, JHEP 0404 (2004) 078, [hep-ph / 0402175 ].

[7] DUNE Collaboration, R. Acciarri et al., Long-Baseline Neutrino Facility (LBNF) and Deep Underground Neutrino Experiment (DUNE) Conceptual Design Report Volume 2: The Physics Program for DUNE at LBNF, arXiv:1512.06148.

[8] Hyper-Kamiokande working group Collaboration, T. Ishida, T2HK: J-PARC upgrade plan for future and beyond T2K, arXiv:1311.5287.

[9] Hyper-Kamiokande proto- Collaboration, K. Abe et al., Physics Potentials with the Second Hyper-Kamiokande Detector in Korea, arXiv: 1611.06118.

[10] ESSnuSB Collaboration, E. Baussan et al., A Very Intense Neutrino Super Beam Experiment for Leptonic CP Violation Discovery based on the European Spallation Source Linac: A Snowmass 2013 White Paper, arXiv:1309.7022.

[11] Y.-F. Li, Overview of the Jiangmen Underground Neutrino Observatory (JUNO), arXiv:1402.6143.

[12] Hyper-Kamiokande Collaboration, Hyper-Kamiokande Design Report, KEK-PREPRINT-2016-21，ICRR-REPORT-701-2016-1.

[13] ICAL Collaboration, S. Ahmed et al., Physics Potential of the ICAL detector at the India-based Neutrino Observatory (INO), arXiv:1505.07380.

[14] IceCube-PINGU Collaboration, M. Aartsen et al., Letter of Intent: The Precision IceCube Next Generation Upgrade (PINGU), arXiv:1401.2046.

[15] J. Cao et al., Muon-decay medium-baseline neutrino beam facility, Phys. Rev. ST Accel. Beams 17 (2014) 090101, [arXiv:1401.8125].

[16] J. M. Conrad and M. H. Shaevitz, Multiple Cyclotron Method to Search for CP Violation in the Neutrino Sector, Phys. Rev. Lett. 104 (2010) 141802, [arXiv: 0912.4079 ].

[17] M. Ghosh, P. Ghoshal, S. Goswami, and S. K. Raut, Synergies between neutrino oscillation experiments: an 'adequate configuration for LBNO, JHEP 1403 (2014) 094, [arXiv:1308.5979].

[18] V. Barger, A. Bhattacharya, A. Chatterjee, R. Gandhi, D. Marfatia, and M. Masud, Configuring the Long-Baseline Neutrino Experiment, Phys. Rev. D89 (2014), no. 1 011302, [arXiv: 1307.2519 ].

[19] A. Ghosh, T. Thakore, and S. Choubey, Determining the Neutrino Mass Hierarchy with INO, T2K, NOvA and Reactor Experiments, JHEP 1304 (2013) 009, [arXiv : 1212.130 5].

[20] W. Winter, Neutrino mass hierarchy determination with IceCube-PINGU, Phys.Rev. D88 (2013) 013013, [arXiv:1305.5539].

[21] M. Ghosh, S. Goswami, and S. K. Raut, Maximizing the DUNE early physics output with current experiments, Eur. Phys. J. C76 (2016), no. 3 114, [arXiv: 1412.1744 ]. 
[22] S. K. Agarwalla, M. Ghosh, and S. K. Raut, A hybrid setup for fundamental unknowns in neutrino

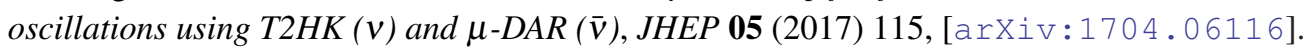

[23] S. K. Raut, Matter effects at the T2HK and T2HKK experiments, Phys. Rev. D96 (2017), no. 7 075029, [arXiv:1703.07136].

[24] M. Ghosh, P. Ghoshal, S. Goswami, N. Nath, and S. K. Raut, New look at the degeneracies in the neutrino oscillation parameters, and their resolution by T2K, NOVA and ICAL, Phys. Rev. D93 (2016), no. 1 013013, [arXiv:1504.06283].

[25] S. K. Agarwalla, P. Huber, J. M. Link, and D. Mohapatra, A new approach to anti-neutrino running in long baseline neutrino oscillation experiments, JHEP 04 (2011) 099, [arXiv : 1005 . 4055 ].

[26] S. K. Agarwalla, S. Choubey, and S. Prakash, Probing Neutrino Oscillation Parameters using High Power Superbeam from ESS, JHEP 12 (2014) 020, [arXiv: 1406 . 2219].

[27] M. Blennow, P. Coloma, and E. Fernández-Martinez, The MOMENT to search for CP violation, JHEP 03 (2016) 197, [arXiv: 1511.02859 ].

[28] S. K. Agarwalla, S. S. Chatterjee, and A. Palazzo, Octant of $\theta_{23}$ in danger with a light sterile neutrino, Phys. Rev. Lett. 118 (2017), no. 3 031804, [arXiv: 1605.04299 ].

[29] M. Ghosh, S. Gupta, Z. M. Matthews, P. Sharma, and A. G. Williams, Study of parameter degeneracy and hierarchy sensitivity of NOVA in presence of sterile neutrino, Phys. Rev. D96 (2017), no. 7 075018, [arXiv:1704.04771].

[30] S. Choubey, D. Dutta, and D. Pramanik, Imprints of a light Sterile Neutrino at DUNE, T2HK and T2HKK, Phys. Rev. D96 (2017), no. 5 056026, [arXiv: 1704.07269 ].

[31] M. Blennow, S. Choubey, T. Ohlsson, and S. K. Raut, Exploring Source and Detector Non-Standard Neutrino Interactions at ESSvSB, JHEP 09 (2015) 096, [arXiv: 1507 . 02868].

[32] P. Coloma, Non-Standard Interactions in propagation at the Deep Underground Neutrino Experiment, JHEP 03 (2016) 016, [arXiv: 1511 . 06357].

[33] J. Liao, D. Marfatia, and K. Whisnant, Nonstandard neutrino interactions at DUNE, T2HK and T2HKK, JHEP 01 (2017) 071, [arXiv: 1612.01443$].$ 